\title{
Aerodynamic Optimization of a Golf Driver Using Computational Fluid Dynamics
}

\author{
Kevin J. Neitzel* \\ Smart Blue Innovations LLC, Wichita, KS, 67207, USA \\ Kyle M. Hanquist ${ }^{\dagger}$ \\ University of Michigan, Ann Arbor, MI, 48109, USA
}

\begin{abstract}
Driving distance and accuracy are the two key characteristics to an ideal golf drive. Besides having correct swing mechanics, there are numerous approaches that have been advanced to improve driver distance and accuracy, including driver shape, size, and material throughout the history of golf. Currently, with strict equipment conformity regulations from the United States Golf Association (USGA), the shape of the golf driver is greatly bounded, resulting in designs with marked improvements in design performance becoming less common. The required blunt body shape of the golf driver leads itself to be highly affected by aerodynamic forces, specifically pressure and viscous drag. Although the general shape of the golf driver head is greatly defined, small changes in shape can affect the aerodynamics significantly. This paper focusing on using Navier-Stokes computational fluid dynamic (CFD) simulations to reduce the aerodynamic drag while also increasing the yaw stability of the golf driver. Results include a characterization of the flowfield experienced during a golf swing as well as the drag analysis of a generic driver. The adjoint method is used to identify surfaces on the driver that are most sensitive to drag. Finally, an optimization approach is discussed to create a low-drag, stable driver with design constraints such as USGA conformity and other parameters important to driver design such as a low center-of-mass and high moment-of-inertia.
\end{abstract}

\section{Nomenclature}

$C_{D} \quad$ Drag Coefficient

$\mathrm{J} \quad$ Objective

$p \quad$ Pressure

$\rho \quad$ Density

$\tau \quad$ Shear Stresses

$\vec{u} \quad$ Velocity vector

$\mathrm{V} \quad$ Volume [cubic centimeters]

$\tilde{x} \quad$ Design Variables

\section{Introduction}

C OLF and aerospace, specifically, aerodynamics have historically been intertwined. From dimples being Tadded to a golf ball to help keep the flow connected to reduce pressure drag to a television show ${ }^{1}$ being focused to the affect of aerodynamics on golf ${ }^{a}$ to Alan Shepard hitting a golf ball on the moon, the sport and the science has been connected. Golf, by it's nature, is a very equipment orientated sport. Ever since the sport was first developed in the middle ages ${ }^{2}$ through when it was advanced into more of how it is

*Founder, Smart Blue Innovations LLC, Wichita, KS, 67207, USA, AIAA Student Member.

${ }^{\dagger} \mathrm{PhD}$ Candidate, Department of Aerospace Engineering, Ann Arbor, MI, 48109, USA, AIAA Student Member.

${ }^{a}$ This paper briefly describes the aerodynamic design from the show. Results are limited based on confidentiality. 
known today, ${ }^{\mathrm{b}}$ the clubs have been innovated to both improve their aesthetics and functions. ${ }^{3}$ Each club has it's own purpose and function, from putters that are used to hit the ball only a few feet or even inches to drivers that hit the ball hundreds of yards. More details of each club type can be found in Ref. 4. However, this paper will focus on only one club called the driver and the club's general purpose is to hit it as far as possible in the intended direction (e.g. fairway). Given that distance and accuracy are generally the two key characteristics to an ideal golf drive. Longer drives result in less distance to the hole on the next shot that will result in the golfer using a higher lofted iron, which is statistically hit with higher accuracy than lower lofted irons. ${ }^{5}$ The distance of the drive depends directly on the club head speed at impact. Previous studies suggest that the distance of the drive will increase by two to three yards for every increase in one mph. ${ }^{6}$ The club head can be increased by improving swing mechanics (e.g. golfer improves golf swing) or by improving the efficiency of the equipment in which this paper will focus on. The three current approaches for increasing club head speed through equipment (i.e. driver) improvement include: 1) reducing the drag of the club during swing, 2) reducing the club weight, and 3) extending the length of shaft. The most popular approach is to reduce the drag of the club during the swing due to rule restrictions ${ }^{7}$ in approaches 2 and 3. Since the driver is a blunt body shape by its nature, it is very susceptible to aerodynamic forces, specifically pressure drag. While the general shape of the club is constrained by the USGA, approaches developed in the aerospace industry, such as the adjoint method, ${ }^{8}$ can be applied to identify sensitive areas of the surface to pressure drag. As been shown in the aerospace field, fine-tuning these sensitive areas can lead to significant reductions in drag. ${ }^{9}$

This manuscript will focus on reducing the aerodynamic drag while increasing the yaw stability of a golf driver utilizing Navier-Stokes computational fluid dynamic (CFD) simulations. With recent progresses in CFD, ${ }^{10}$ it can identify areas of improvement that would require many wind tunnel experiments such as shown in Fig. 1. First, the design approach and methodology to golf driver design will be briefly presented. Then the numerical approach will be discussed that details the numerical tools and implementations used to model the golf driver. The flowfield is then characterized during a typical golf swing. The adjoint method is used to identify surfaces on the driver that are most sensitive to drag. Finally, an optimization approach is discussed to create a low-drag, stable driver while with design constraints such as USGA conformity and other parameters important to driver design such as a low center-of-mass and high moment-of-inertia. The optimization approach is similar to optimization's done in the aircraft field. ${ }^{11,12}$ The design methodology achieved a globally optimal design with a practical level of computational effort. The paper concludes with conclusions and future work recommendations.

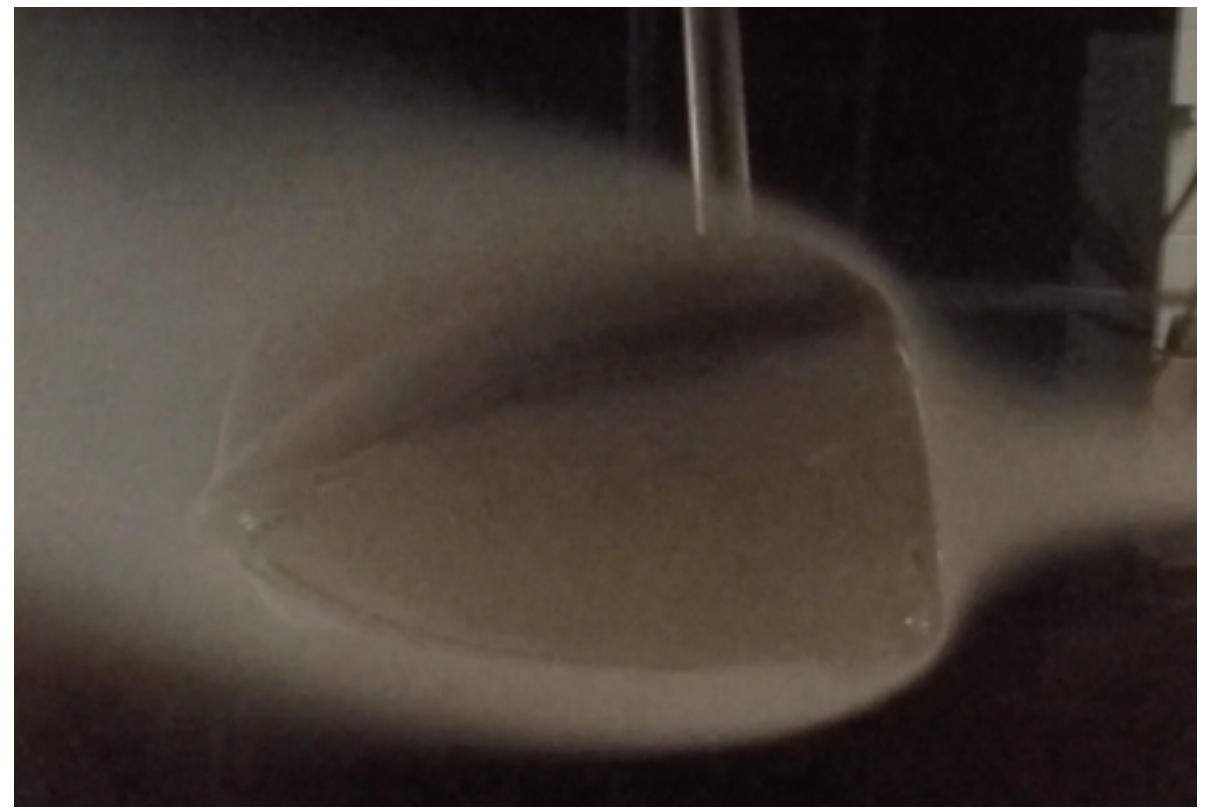

Figure 1: Golf driver head in University of Michigan subsonic wind tunnel

\footnotetext{
${ }^{\mathrm{b}}$ Modern golf commonly credited to beginning at England's St. Andrew's golf course in the 18th century.
} 


\section{Design}

\section{II.A. Motivation}

Golf driver design is a complex, multi-disciplinary task. The final goal of the golf driver is to be able to hit the golf ball in the fairway with the most distance possible. Golf club design has evolved substantially through the history of the game and the golf driver design has particularly changed in the last 30 years with the introduction of metal construction. This recent evolution was driven by manufacturing processes and new metal alloys. Figure 2 presents the recent evolution of the golf driver design. The growth of the golf driver can be attributed to pursuit of higher coefficient of restitution for the club face (e.g. trampoline effect) and higher moment of inertia (MOI). The results of these two attributes are faster ball speeds and a more stable clubhead through impact.

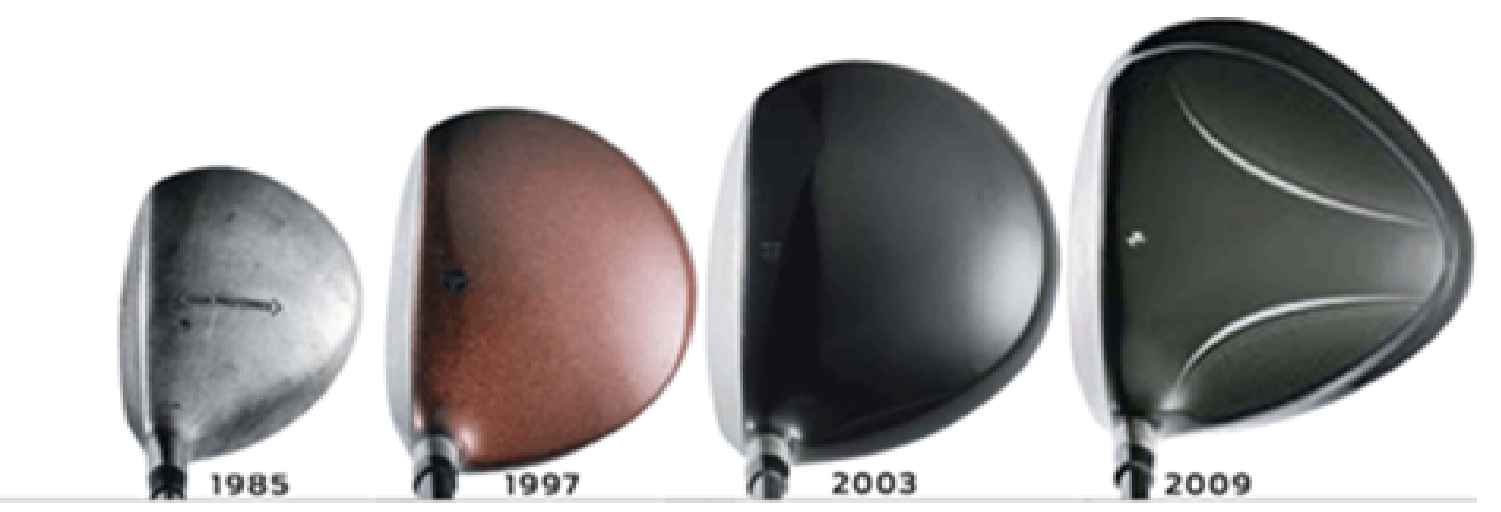

Figure 2: Evolution of Golf Driver Size ${ }^{13}$

In the 2000's, the USGA wanted to regulate the constantly increasing size of the golf driver and the "trampoline effect". The USGA introduced rules to limit the volume of the clubhead and the coefficient of restitution (COR) of the face. ${ }^{7}$ The USGA limitation on clubhead volume and face COR have pushed manufacturers to look for performance gains in other areas. Recently, aerodynamics has come to the forefront of research and development for golf manufacturers. Figure 3 presents examples of manufacturers pursuing improvements in aerodynamic performance.

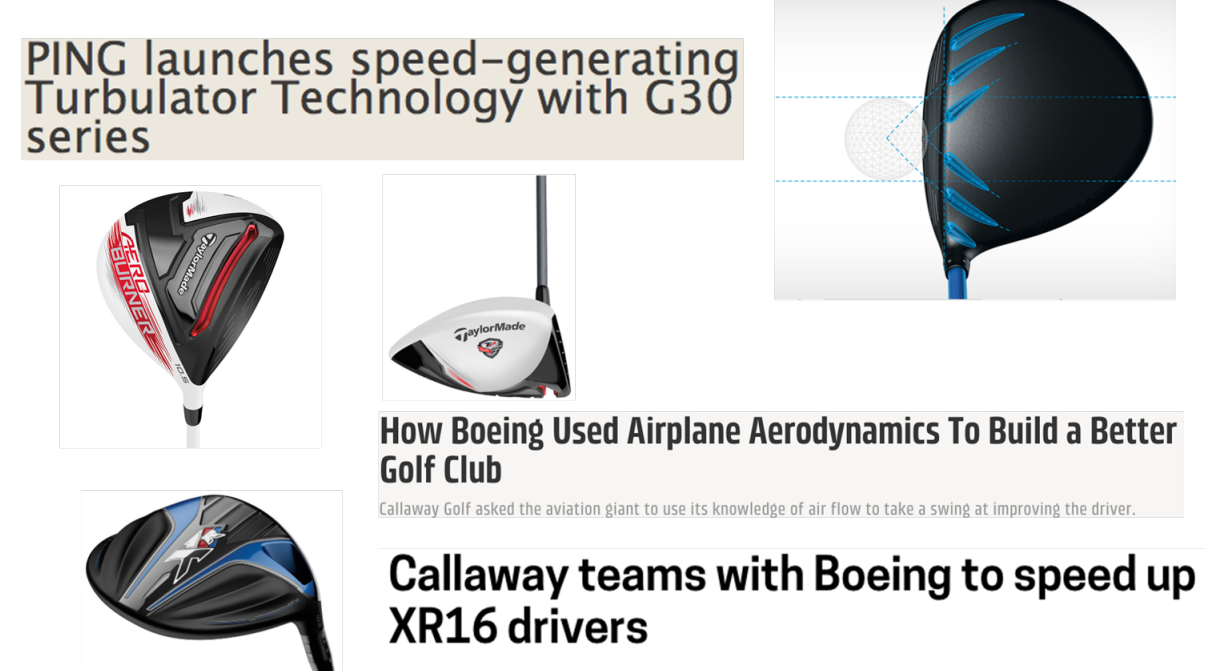

XR16, XR16 Pro drivers emphasize forgiveness and aerodynamics

Figure 3: Trend Toward Aerodynamics

In order to develop a systematic design process, design objectives and constraints must be clearly defined. The present work aims to deliver maximum distance with superior forgiveness. Distance depends on many factors including clubhead speed, coefficient of restitution, and clubhead orientation. Forgiveness is how well 
the clubhead performs under non-optimal conditions. The rules of golf provide some relief in the design effort. ${ }^{7}$ The face coefficient of restitution is governed and cannot exceed 0.83 in value. Additionally, the golf driver cannot exceed 460cc in volume. Golf club manufacturers have developed design guidelines to meet the COR and volume limit. The critical design element for meeting these limitations is the face design, specifically, the face size and material thickness. The present work will adopt the industry standards for the face size, material thickness, and 460cc volume. These parameters will be held constant.

The main focus and design objectives for this effort will be the aerodynamic drag and aerodynamic stabilization. These objectives correspond to the goals of more distance through a higher clubhead speed and more forgiveness by being aerodynamically stable to correct for errors in clubhead orientation. The errors in club orientation about any axis can happen. However, the dominate axis of error is about the shaft axis. This would be analogous to the yaw degree of freedom for an airplane. The present work will focus only on aerodynamic drag, where the results for aerodynamic stability, specifically yaw stability are discussed in Refs. 1 and 15 .

\section{II.B. Methodology}

As described, the design effort will focus only on aerodynamic shaping. The objectives will be drag and yaw stability. Each of these objectives will be evaluated at a single condition during the swing that corresponds to the impact zone. This is when the club head is aligned with to the flowfield (ideally) and at the highest speed during the swing. Since the aerodynamic forces scale with the square of the velocity, the impact zone is the appropriate choice for the design work. The optimization of the club head design will use a multi fidelity approach. First, a coarse design selection will be completed using a 3 parameter representation of a club head (plan form width, platform depth, and beltline height). Second, the best design from the coarse design step will be used as the starting point for the final, detailed surface optimization. The coarse design step will be a simple selection process based on a parameter space survey. The final design step will be a sophisticated, adjoint driven surface optimization. The multi fidelity approach will ensure an optimal shape is achieved with a practical level of computational expense. Large deformations of the surface that would take a long time to achieve are captured with a three parameter representation. Small surface changes will be handled by the adjoint based surface optimization.

\section{Numerical Approach}

The CFD code used in this work is SU2, which was developed at Stanford University and is open-source. ${ }^{14}$ The CFD analysis solves the Navier-Stokes equations shown below. The flow is considered turbulent and the Spalart-Allmaras turbulence model is employed.

$$
\begin{gathered}
\frac{\partial \rho}{\partial t}+\vec{\nabla} \cdot(\rho \vec{u})=0 \\
\frac{\partial(\rho \vec{u})}{\partial t}+\vec{\nabla} \cdot[\rho \overline{\overline{u \otimes u}}]=-\overrightarrow{\nabla p}+\vec{\nabla} \cdot \overline{\bar{\tau}}+\rho \vec{f} \\
\frac{\partial(\rho e)}{\partial t}+\vec{\nabla} \cdot((\rho e+p) \vec{u})=\vec{\nabla} \cdot(\overline{\bar{\tau}} \cdot \vec{u})+\rho \vec{f} \vec{u}+\vec{\nabla} \cdot(\overrightarrow{\dot{q}})
\end{gathered}
$$

The CFD analysis is used to characterize the aerodynamic behavior of the geometry. Additionally, the adjoint method is used to determine the surface sensitivity relative to an objective function. The objective function for this work is the club head drag. The surface sensitivity metric will be used to drive the design optimization for the club head.

\section{III.A. Simulation Setup}

The golf club experiences a range of speeds and angle of attacks during a typical swing. However, since the aerodynamic forces are proportional to the velocity squared, the test cases will only be run at the conditions where the golf head speed is the fastest, which is at impact with the golf ball in order to save computational cost. This is a common approach in golf club design. ${ }^{6}$ The speeds of interest are from $80 \mathrm{mph}$ (average golfer) to $130 \mathrm{mph}$ (long drive professional). ${ }^{16}$ This is a wide range of velocities, however it will be shown that the aerodynamic behavior is Reynolds number independent. The swing speed of $100 \mathrm{mph}$ is used in the 
presented work. The design simulation setup is limited to the body of the club head. Changes to the club face would not be viable. Additionally, geometric constraints are applied that correspond to the governing rules of golf clubs. The width and depth dimensions are limited and the depth can not be larger than the width. These constraint act as boundaries of the design space allowable for the golf driver geometry.

\section{Results}

\section{IV.A. Golf Driver Flow Behavior}

The golf driver flow behavior is dictated by the geometric constraints of golf club design. The driver must have a large flat surface on the leading side of the club in order to make consistent contact with the golf ball. Additionally, the body of the driver is constraint by two major rules set by golf's sanctioning body, the United States Golf Association (USGA). First, the overall volume of the golf driver is limited to a maximum of $460 \mathrm{cc}$. Second, the width (side-side) dimension of the club head must be equal to or larger than the depth (fore-aft) dimension. These geometric constraints lead to a bluff body geometry.

Figure 4 shows the velocity contours over a typical golf driver head geometry. The flow approaches the club head and stagnates at the face of the driver. The flow then has to make a quick turn around the transition between the driver face and the club body. The flow is able to stay attached around the bottom and sides of the club. However, the flow across the transition to the crown of the club separates. As mentioned previously, the club head is a bluff body so flow separation is not unusual. The golf driver's aerodynamic behavior, like most bluff bodies, is pressure dominated. The low speed, low pressure region of separated flow is the primary contributor to aerodynamic drag. It should be noted that the club face also contributes significantly to drag. However, this portion of the geometry can not be changed. The golf club must have a large flat club face to be a viable design.

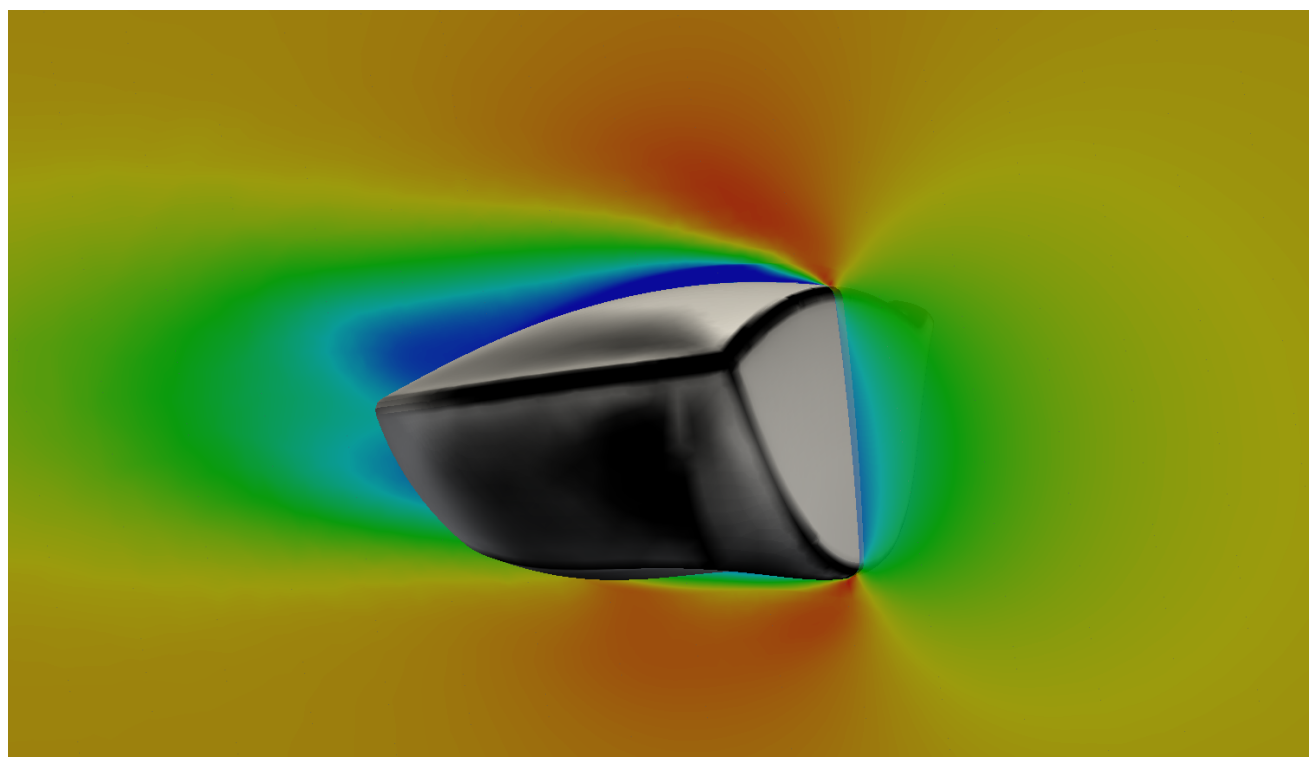

Figure 4: Velocity contours over typical golf driver head

Figure 5 highlights the flow separation by presenting the turbulent eddy viscosity. The flow behaves better on the bottom side of the club, but the crown of the club is predominately separated. Intuition suggests that reducing the flow separation around the club head will be the best way to reduce the drag. 


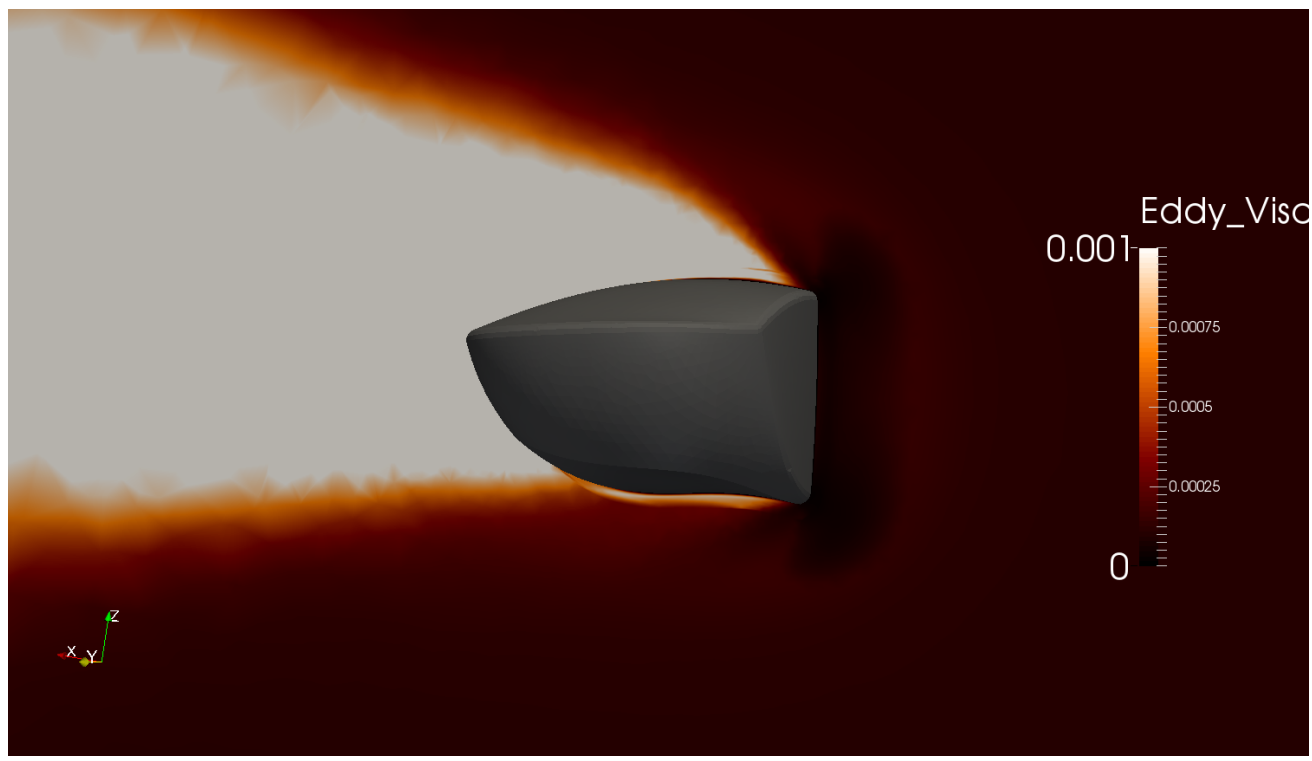

Figure 5: Aerodynamic drag sensitivity on typical golf driver head

Next, the adjoint solution is presented. The adjoint objective is drag for the club head. The blue regions correspond to surfaces that should be pushed in to reduce drag, while red regions should be pulled out to reduce the drag. Figure 6 shows that the adjoint solver is acting to soften the transition radius between the face and the crown. This shape modification is directly addressing the flow separation on the crown. The suggested softening will suppress, if not stop, the crown flow separation. This particular observation from the adjoint solution is quite intuitive and would have been obvious from the flow solution. However, the adjoint solution is truly powerful in the subtle suggestions that are not obvious in the flow solution as can be seen throughout the surface of driver.

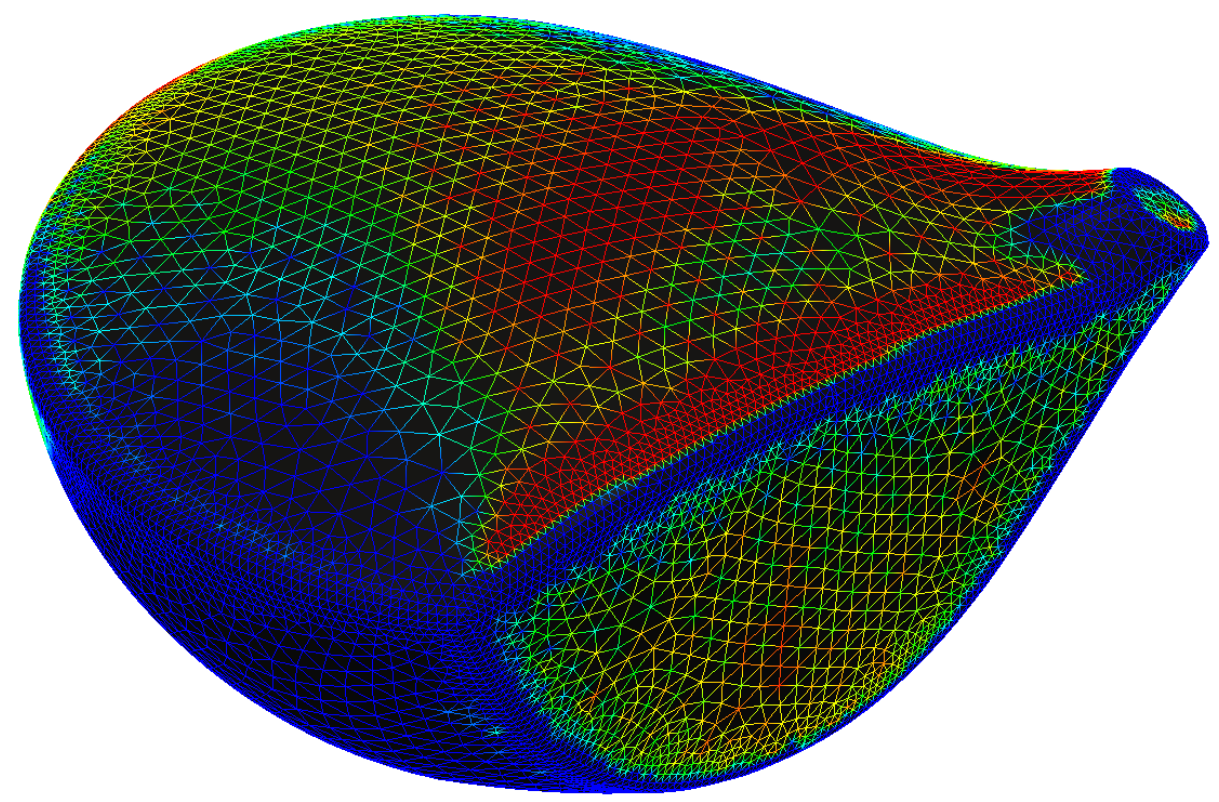

Figure 6: Drag Reduction Sensitivity: Isometric View (blue: push in, red: pull out) 
Figure 7 shows a side view of the surface sensitivity. This particular plots shows some surface changes that are not obvious from the flow solution. Specifically, the toe portion of the club and the bottom sole of the club. Subtle changes are suggested by the adjoint to reshape the toe and reduce the sole cavity slightly. The design optimization scheme utilizes the surface sensitivities provided by the adjoint solution. ${ }^{\mathrm{c}}$

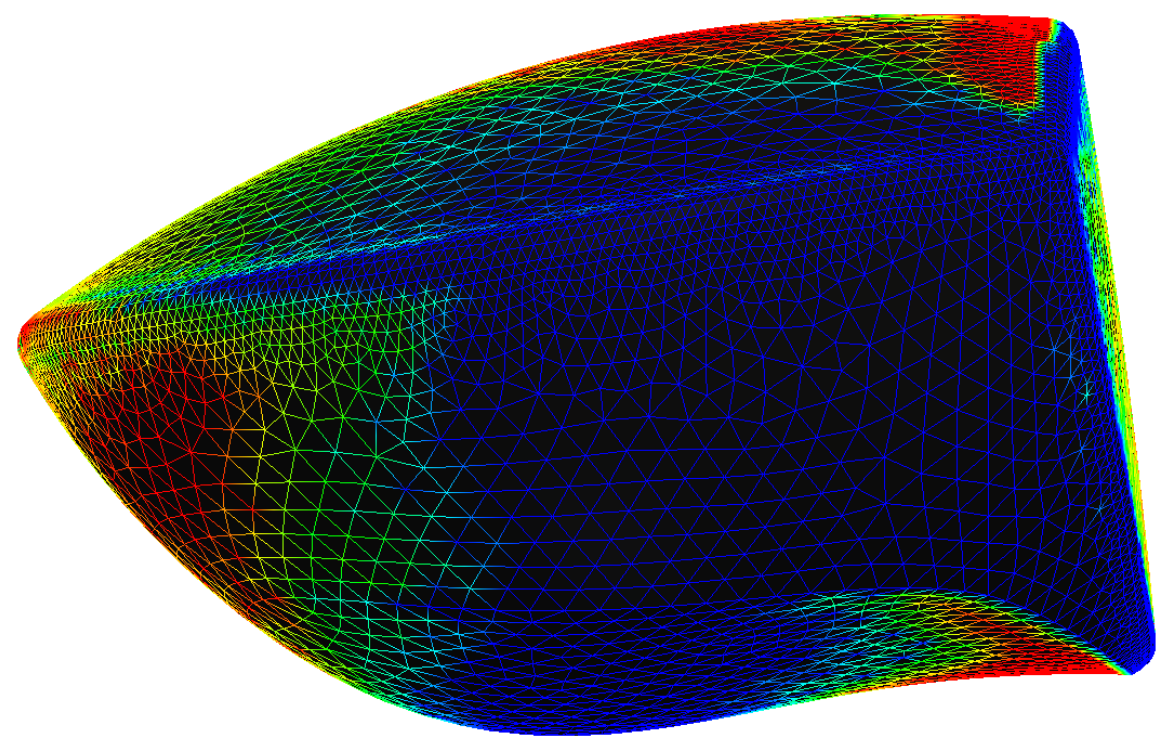

Figure 7: Drag Reduction Sensitivity: Side View (blue: push in, red: pull out)

\section{Conclusions}

The design space of a modern day golf driver is briefly discussed and explored. The design methodology aims to reduce the aerodynamic drag of the club head to help the golfer swing the club faster, leading to longer drives. The aerodynamic performance of the golf driver is evaluated using CFD. Additionally, the adjoint methodology is applied in order to understand the surface sensitivity with respect to a given objective, in this case, drag. The adjoint surface sensitivities have been used in a design optimization scheme to develop a low drag driver geometry. While some drag inducing surfaces of the driver resulting in drag are constrained, small changes such as softening the face to crown radius can result in significant reduction in drag. Although the details are limited due to confidentiality, small changes to the driver shape can result in over $60 \%$ reduction in aerodynamic drag compared to a generic driver. The overall design methodology has proven that significant drag reduction can be achieved, while still maintaining the core functionality and geometric rule constraints of a golf driver. More details of the work are discussed in Ref. 15.

\footnotetext{
${ }^{\mathrm{c}}$ Due to confidentiality, results and figures are limited. However, results will be posted to Ref.15 as they become available.
} 


\section{References}

${ }^{1}$ Ponti, J. and Valle, A., "Computational Fluid Dynamics", Driver vs. Driver, Golf Channel, Orlando, 2016.

${ }^{2}$ Browning, R., A History of Golf: The Royal and Ancient Game, E. P. Dutton \& Company, New York, 1955.

${ }^{3}$ Green, A, "A Brief History of the Golf Club," Popular Mechanics, May 2013. [http://www.popularmechanics.com/adventure/sports/a8984/a-brief-history-of-the-golf-club-15478412. Accessed 12/1/16.]

${ }^{4}$ Lindell, J. "Types of Golf Clubs \& Their Uses", Golfsmith, January 2016. [http://blog.golfsmith.com/golfequipment/types-of-golf-clubs-their-uses. Accessed 12/1/16.]

${ }^{5}$ Hume, A. H., Keogh, J., and Reid, D., "The Role of Biomechanics in Maximising Distance and Accuracy of Golf Shots," Review Article, Sports Medicine, Vol. 35, 2005, pp. 429-449.

${ }^{6}$ Henrikson, E., Wood, P., and Hart, B., "Experimental Investigation of Golf Driver Club Head Drag Reduction Through the Use of Aerodynamic Features on the Driver Crown," Procedia Engineering, Vol. 72, 2014, pp. 726-731.

${ }^{7}$ Rules of Golf, USGA, 2016.

${ }^{8}$ Jameson, A., "Optimum Aerodynamic Design Using CFD and Control Theory," AIAA Paper 1995-1729, 1995.

${ }^{9}$ Giannakoglou, K. C., "Design of Optimal Aerodynamic Shapes Using Stochastic Optimization Methods and Computational Intelligence", Progress in Aerospace Sciences, Vol. 38, No. 1, 2002, pp. 43-76.

${ }^{10}$ Fujii, K., "Progress and Future Prospects of CFD in a AerospaceWind Tunnel and Beyond," Progress in Aerospace Sciences, Vol 41, 2005, pp. 455470.

${ }^{11}$ Palacios, F., Economon, T. D., Wendorff, A. D., and Alonso, J. J., "Large-scale Aircraft Design Using SU2," AIAA Paper 2015-1946, 2015.

${ }^{12}$ Chen, S., Lyu, Z., Kenway, G. K., and Martins, J. R., "Aerodynamic Shape Optimization of Common Research Model WIng-Body-Tail Configuration," Journal of Aircraft, 2015, pp. 1-18.

${ }^{13}$ Herity, J, "Evolution of Improvement," Golf Digest, January 2009. [http://www.golfdigest.com/story/hotlistevolution0902. Accessed 12/1/16.]

${ }^{14}$ Palacios, F., Colonno, M. R., Aranake, A. C., Campos, A., Copeland, S.R., Economon, T. D., Lonkar, A. K., Lukaczyk, T. W., Taylor, T. W. R., and Alonso, J. J., "Stanford University Unstructured (SU2): An open-source integrated computational environment for multi-physics simulation and design," AIAA Paper 2013-0287, 2013.

${ }^{15}$ Neitzel, K. J. and Hanquist, K. M., "Aerodynamics and Golf", Smart Blue Innovations, Kansas, January 2017. [http://smartblueinnovations.com/golf. Accessed 1/7/17.]

${ }^{16}$ Bowden, J., "Average Swing Speed Chart," Swingman Golf, Octover 2015. [http://www.swingmangolf.com/average-golfswing-speed-chart-2. Accessed 6/1/16.] 